\title{
Schwanoma Lingual en un Adolescente: Revisión de la Literatura a Propósito de un Caso
}

\author{
Schwannoma of the Tongue in an Adolescent: \\ A Short Literature Review Apropos of a Case
}

\author{
Rodrigo Badilla*; Rolando Carrasco,"*; Erita Cordero*,"* \& Cristóbal Pacheco"*
}

BADILLA, R.; CARRASCO, R.; CORDERO, E. \& PACHECO, C. Schwanoma lingual en un adolescente: revisión de la literatura a propósito de un caso. Int. J. Odontostomat., 7(2):199-201, 2013.

RESUMEN: El Schwanoma corresponde a un tumor neurogénico benigno, derivado de las células de la cresta neural, específicamente de las células de la vaina periférica nerviosa llamadas schwannocitos. El 25\% de éstos, se presentan en cabeza y cuello, y sólo un $1 \%$ se presentan en la cavidad oral, principalmente en la lengua. Presentamos un caso de una niña de 13 años de edad con diagnóstico de Schwannoma de lengua. El propósito de este reporte es enfatizar que el schwanoma lingual es un diagnóstico diferencial a considerar, pese a su baja prevalencia, entre todas las otras lesiones linguales en niños o adolescentes.

PALABRAS CLAVE: schwanoma, lengua, adolescente.

\section{INTRODUCCIÓN}

El Schwanoma, también denominado neurilemoma o neurinoma, corresponde a un tumor neurogénico benigno, derivado de las células de la cresta neural, específicamente de las células de la vaina periférica nerviosa llamadas schwannocitos (Zachariades et al., 2001). Puede afectar a nervios sensitivos, motores, simpáticos, sensoriales y pares craneales, con excepción de los nervios óptico y olfatorio, que carecen de esta vaina (Thawley et al., 1999). Se caracterizan por ser capsulados, presentar un crecimiento lento y clínicamente asintomáticos. De haber síntomas, dependerán del tamaño y la localización del tumor (Dreher et al., 1997).

Su ubicación es poco común en los nervios periféricos, extracranealmente cerca de un $25 \%$ de los schwanomas están localizados en cabeza y cuello. Sólo el $1 \%$ se presenta en la cavidad oral, principalmente en la lengua, pero también se han descrito en el paladar, mucosa bucal, labios y encía, en orden decreciente (Arda et al., 2003; Bansal et al., 2005).

Se pueden presentar en cualquier edad, sin embargo, son más prevalentes entre la $3^{\mathrm{a}}$ y $6^{\mathrm{a}}$ década de vida, no obstante, en su ubicación bucal se presentan con mayor frecuencia en la $2^{\mathrm{a}}$ y $3^{\mathrm{a}}$ década, sin predilección por sexo y la etiología es aún desconocida (Pfeifle et al., 2001).

Histológicamente, se observa encapsulado y posee un patrón de distribución celular característico, conocido como Antoni A (hipercelularidad, distribución de células en empalizada, conocidos como "cuerpos de Verocay") y Antoni B (hipocelularidad, células desordenadas e inmunohistoquímicamente positiva a proteínas S100 y vimentina) (Pfeifle et al.).

\section{REPORTE DEL CASO}

Se presenta una paciente de sexo femenino, de 13 años de edad, que consulta al servicio de cirugía maxilofacial del Complejo Hospitalario San Borja Arriarán, derivada por el servicio de cirugía pediátrica del Hospital Felix Bulnes Cerda con diagnóstico de tumor lingual.

\footnotetext{
* Servicio de Cirugía Maxilofacial, Hospital Clínico San Borja Arriarán, Santiago, Chile.

"Facultad de Odontología, Universidad de Chile, Santiago, Chile.
} 
En relación a la historia médica, la paciente no refiere antecedentes mórbidos relevantes. En el examen físico general y segmentario de cabeza y cuello, no se encuentran signos patológicos. Al examen bucal se observa un aumento de volumen en el dorso lingual, de aproximadamente $1,5 \mathrm{~cm}$ de diámetro, firme, recubierto por mucosa normal, no adherido a planos profundos e indoloro.

Se solicitaron estudios complementarios como ecografía doppler, hemograma, tiempo de protrombina, INR y grupo RH. Con la hipótesis diagnóstica de lipoma lingual, se realizó la biopsia excisional. La técnica quirúrgica consistió en una incisión en la mucosa sobre la lesión y debridación de los planos del tumor de los tejidos periféricos (plano de clivaje adecuado) para lograr la exéresis completa de la lesión (Figs. 1 y 2). Posteriormente a la hemostasia se realizó un cierre por primera intención.

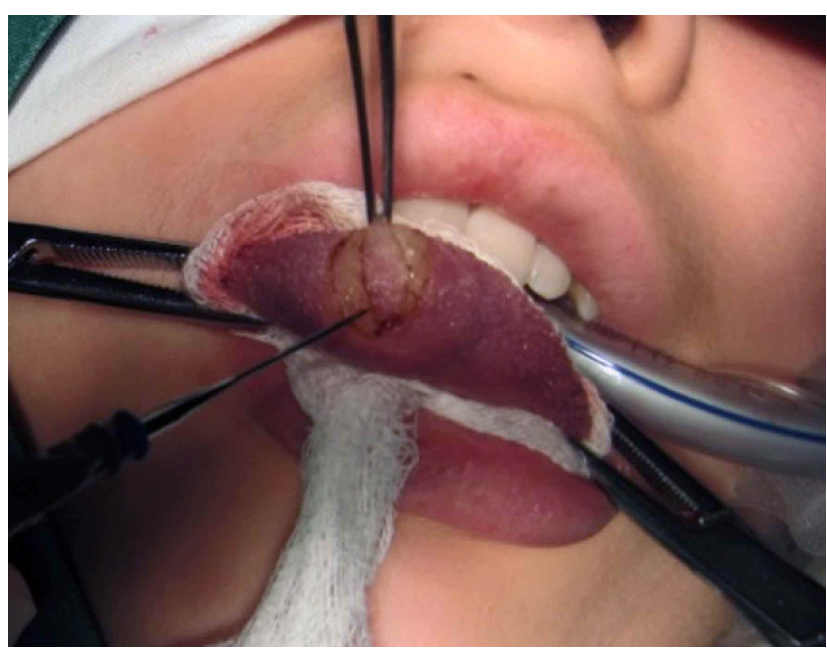

Fig. 1. Exéresis de la lesión.

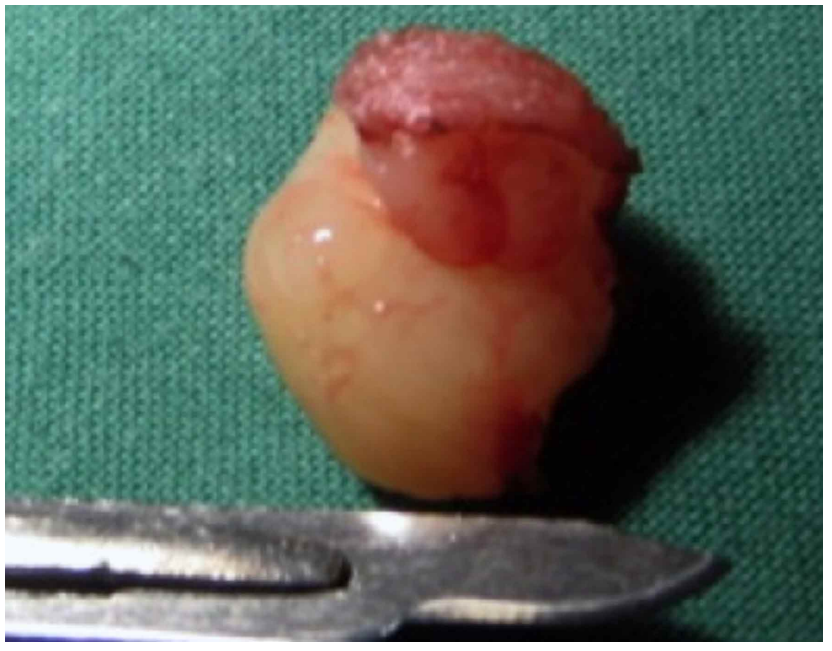

Fig. 2. Pieza quirúrgica resecada.
No se presentaron complicaciones quirúrgicas pre o post operatorias. El tejido fue enviado a estudio histopatológico. El diagnóstico histológico de la lesión fue compatible con Schwanoma. Desde abril del año 2009, fecha de la intervención quirúrgica, no se evidencian signos de recurrencia.

\section{DISCUSIÓN}

Los tumores de nervios periféricos en la cavidad oral corresponden a schwanomas, neurofibromas y neuromas traumáticos principalmente. El schwanoma corresponde a una tumoración benigna producto de la proliferación de los schwannocitos de la vaina neural. Aproximadamente un $50 \%$ de los hallazgos de estos tumores en la cavidad oral, tienen una relación directa con un nervio, en el caso de la lengua, fundamentalmente de los nervios glosofaríngeo, hipogloso o lingual (Naidu \& Sinha, 2010).

Para el diagnóstico diferencial se deben tener presentes las patologías benignas como el lipoma, fibroma traumático, leiomioma, tumor de células granulares, neuroma y adenoma, pero también se debe tener presente la posible presencia de tumores malignos como sarcomas (Gallesio \& Berrone, 1992; Karaca et al., 2010). Los schwanomas suelen ser solitarios y es por ello que en compañía de otras lesiones similares debe sospecharse de neurofibromas y por consiguiente descartar una posible neurofibromatosis (Luksic et al., 2011).

Los estudios complementarios son de ayuda para establecer el diagnóstico presuntivo y dependiendo de la ubicación podrán utilizarse desde la ecografía hasta resonancia nuclear magnética (RNM) (de considerarlo la clínica necesario). La RNM puede mostrar no sólo el tumor y su cápsula, sino también, en determinadas casos, el nervio a partir del cual se desarrolla (Ku \& Yeh, 2000). Sin embargo, el diagnóstico definitivo es histopatológico (Hsu et al., 2006).

Aunque los schwanomas son usualmente asintomáticos, si no son tratados oportunamente, la ubicación lingual podría generar problemas como disfagia, dislalia, disnea y apnea obstructiva del sueño en casos extremos (Karaca et al.).

El tratamiento del schwanoma es la excisión quirúrgica completa de la lesión (Zachariades et al., 2001), por lo que la recurrencia generalmente obedece a una 
exéresis incompleta. No hay reportes de malignización de schwanomas (Mevio et al., 2002).

Los schwanomas que aparezcan en troncos nerviosos mayores, deberán ser disecados sin dañar el nervio, debido a que las fibras nerviosas no forman parte del tumor, que surge de la envoltura del nervio y desplaza a los axones hacia un lado (Thawley et al.; Miyawaki et al., 1999). Tras la extirpación de este tumor benigno pueden producirse secuelas neurológicas transitorias o permanentes, por lo que se debe informar, previa cirugía, sobre esta posibilidad (Miyawaki et al.; de Vicente et al., 2003).
Cuando se ubican en la lengua, el tratamiento quirúrgico debe ser cuidadoso, debido a que podría generarse gran perdida de masa lingual lo que podría llegar a producir problemas en la fonación y deglución (Karaca et al.).

El reporte de nuestro caso clínico resulta muy interesante, debido a que los schwanomas presentan una baja prevalencia en niños y adolescentes, y nos da una referencia de las posibles patologías que debemos tomar en cuenta al momento de establecer la hipótesis diagnóstica de tumores linguales en pacientes de corta edad.

BADILLA, R.; CARRASCO, R.; CORDERO, E. \& PACHECO, C. Schwannoma of the tongue in an adolescent: a short literature review apropos of a case. nt. J. Odontostomat., 7(2):199-201, 2013.

ABSTRACT: A schwannoma is a benign neurogenic tumour originating from schwannocyte of the nerve sheat. A $25 \%$ of all schwannomas are seen in the tissues of the head and neck and only a $1 \%$ of them are located in oral cavity, especially in tongue. We report the case of a 13 year old girl diagnosed with a schwannoma of the tongue. The purpose of this report is to empathize that schwannoma of the tongue is a differential diagnostic to consider among all other lingual lesions in children or adolescents.

KEY WORDS: neurilemmoma, tongue, adolescent.

\section{REFERENCIAS BIBLIOGRÁFICAS}

Arda, H. N.; Akdogan, O.; Arda, N. \& Sarikaya, Y. An unusual site for an intraoral schwannoma: a case report. Am. J. Otolaryngol., 24(5):348-50, 2003.

Bansal, R.; Trivedi, P. \& Patel, S. Schwannoma of the tongue. Oral Oncol. Extra., 41(2):15-7, 2005.

de Vicente Rodríguez, J. C.; Junquera Gutiérrez, L. M.; Fresno Forcelledo, M. F. \& López Arranz, J. S. Neck Schwannomas. Med. Oral, 8(19):71-6, 2003.

Dreher, A.; Gutmann, R. \& Grevers, G. Extracranial schwannoma of the ENT region. Review of the literature with a case report of benign schwannoma of the base of the tongue. HNO, 45(6):468-71, 1997.

Gallesio, C. \& Berrone, S. Schwannoma located in the tongue. A clinical case report. Minerva Stomatol., 41(12):583-90, 1992.

Hsu, Y. C.; Hwang, C. F.; Hsu, R. F.; Kuo, F. Y. \& Chien, C. Y. Schwannoma (neurilemmoma) of the tongue. Acta Otolaryngol., 126(8):861-5, 2006.

Karaca, C. T.; Habesoglu, E. T.; Naiboglu, B.; Habesoglu, M.; Oysu, C.; Egeli, E.; et al. Schwannoma of the tongue in a child. Am. J. Otolaryngol., 31(1):46-8, 2010.

Ku, H. C. \& Yeh, C. W. Cervical schwannoma: a case report and eight years review. J. Laryngol. Otol., 114(6):414-7, 2000.

Luksic, I.; Müller, D.; Virag, M.; Manojlovic, S. \& Ostovic, K.
Schwannoma of the tongue in a child. J. Craniomaxillofac. Surg., 39(6):441-4, 2011.

Mevio, E.; Gorini, E.; Lenzi, A. \& Migliorini, L. Schwannoma of the tongue: one case report. Rev. Laryngol. Otol. Rhinol. (Bord.), 123(4):259-61, 2002.

Miyawaki, T.; Nakamura, A.; Hayashi, A. \& Kurihara, K. Macrocystic Schwannoma in the seventh cervical nerve. Plast. Reconstr. Surg., 104(3):789-92, 1999.

Naidu, G. S. \& Sinha, S. M. Schwannoma of the tongue: an unusual presentation. Indian J. Dent. Res., 21(3):457-9, 2010 .

Pfeifle, R.; Baur, D. A.; Paulino, A. \& Helman, J. Schwannoma of the Tongue: Report of 2 cases. J. Oral Maxillofac. Surg., 59(7):802-4, 2001.

Thawley, S.; Panje, W.; Batsakis, G. \& Lindberg, R. Tumors of the neck. Philadelphia, WB Sanders Co., 1999. pp.133840.

Zachariades, N.; Skoura, C.; Papageorgiou, G. \& Chrissomali, E. Giant ancient neurilemmoma of the cervical region: Report of Case. J. Oral Maxillofac. Surg., 59(6):668-72, 2001.

Dirección para Correspondencia:

Rodrigo Badilla Monasterio

Servicio Cirugía Maxilofacial

Hospital San Borja Arriarán (CDT)

Santa Rosa 1234, Santiago

CHILE

Email: dr.rbadilla@gmail.com
Recibido : 07-12-2012 Aceptado: 28-01-2013 